\title{
Label-Free Peptide-Based Biosensor for Express Detection of Protein Markers of Acute Cardiovascular Conditions in Biological Fluids ${ }^{+}$
}

\author{
Nikita Sitkov *, Tatiana Zimina *, Vladimir Karasev, Olesya Naretskaya and \\ Margarita Kiseleva \\ Micro- and Nanoelectronics, Saint-Petersburg Electrotechnical University “LETI" (ETU), St. Petersburg \\ 197376, Russia; \\ * Correspondence: sitkov93@yandex.ru (N.S.); tmzimina@gmail.com (T.Z.) \\ + Presented at the 1st International Electronic Conference on Biosensors, 2-17 November 2020; \\ Available online: https://iecb2020.sciforum.net/.
}

Received: date; Accepted: date; Published: date

\begin{abstract}
Acute cardiovascular conditions require prompt assistance, which depends on a timely and accurate diagnosis. This could be achieved by using biosensor systems based on peptide aptamers capable of selectively binding protein markers of diseases. In this work, a label-free biosensor system based on fluorimetric registration of the formation of a "peptide aptamer - target protein" complex is considered. It comprises a microfluidic subsystem integrated with arrays of sites with immobilized peptide aptamers, coupled with optical detection system. The clinical sample of the whole blood is loaded into the inlet basin, where the cells are separated and plasma flows into the microfluidic channel for analysis. Peptide aptamers were created using the molecular complement search technique based on the search for systems of conjugated ion-hydrogen bonds in the three-dimensional structures of target proteins. The technology for manufacturing a microfluidic chip is a combination of thick-film and photolithography technologies based on the SU-8 photoresist, for which the relief and surface morphology have been studied. The composition of the biochip layers is selected in such a way that ultraviolet light with a wavelength of $280 \mathrm{~nm}$ passes through inlet window, excites fluorescence inside the channel, which passes through the glass window, which absorbs UV-light. This wavelength accounts for the maximum absorption of aromatic amino acids - tyrosine and tryptophan. In this case, one of the last layers is a luminophore layer for re-emission of protein fluorescence as a visible light. The reading platform includes a 280 nm LED, a video sensor, 3D-printed PLA tooling, and software for processing and analyzing the received signal.
\end{abstract}

Keywords: cardiovascular diseases; label-free detection; peptide aptamers; protein biomarkers; biochips

\section{Introduction}

Currently, cardiovascular diseases are one of the main causes of death in the population. The decisive factor that determines the prognosis of the development of the disease is prompt diagnosis and assistance. Experts note the need for preclinical diagnostic tools. At present time this is mainly electrocardiography, which often does not allow the identification of developing myocardial infarction. It is more reliable to confirm the diagnosis using biochemical methods, namely, by analyzing markers of myocardial infarction in blood. Since traditional means of biochemical diagnostics of such diseases are extremely labor intensive and not sufficiently operative, it seems actual to search ways to create diagnostic tools of a new generation capable of implementing express 
multiparametric diagnostics, and having the potential for development into an expert systems. One of the most promising methods of biochemical express diagnostics is the use of diagnostic microsystems in the form of biosensors or biochips with recognizing elements immobilized on their surface, capable of selectively binding to target marker proteins of diseases.

\section{Materials and Methods}

The main markers used for the biochemical diagnosis of acute myocardial infarction (AMI) are minor serum proteins, such as:

1. Myoglobin;

2. Creatine kinase $\mathrm{MB}(\mathrm{CKMB})$;

3. Cardiac troponin I (cTpI);

4. Cardiac troponin T (cTpT).

Most cardiac biomarkers are distinguished by:

- $\quad$ high concentration in cardiomyocytes;

- content absence or low in other tissues;

- release into the blood in the first hours of AMI, proportional to the amount of damage to cardiomyocytes;

- availability for quantitative determination.

Cardiac troponins have the highest cardiospecificity. Other of the mentioned markers, for example, CKMB and myoglobin, are also present in other tissues (for example, in skeletal muscles) and their increase is less specific in the diagnosis of AMI. In addition, cardiac troponins are the most sensitive among the listed biomarkers, and they enable even a small myocardial damage, that would not have been detected by CKMB determination, to be diagnosed.

Another protein of interest as a biomarker of cardiovascular disorders is myeloperoxidase (MPO). MPO levels increase in blood as a result of an inflammatory process in the arteries, which often causes the destruction of atherosclerotic fat deposits in the vessel wall and subsequent thrombosis. MPO is an enzyme that can cause tissue damage in many inflammatory diseases, in particular cardiovascular diseases. MPO is a very informative marker for monitoring the condition of people who have undergone cardiac surgery, in addition, this marker shows serious metabolic disorders in the body.

To solve the problem of complex quantitative preclinical diagnostics of cardiovascular diseases, multiparametric biochips with a biorecognition elements based on molecular spatial complements, namely, peptide aptamers, could be used.

The development of peptide aptamer sequences could be carried out using various in silico modeling methods [1]. In this work, the modeling was carried out using the "Protein 3D" visualizer of supramolecular structures [2], developed at the Center of Microtechnology and Diagnostics of St. Petersburg State Electrotechnical University. The program enables 3D structures of peptide chains to be visualized and the sites of mutual recognition to be identified. An important feature of the visualizer is the possibility of protein representation in the form of conjugated ion-hydrogen bonds systems (CIHBS), considered as the basis for constructing biostructures and energy transfer channels in these structures. The representation of proteins and peptides in the form of CIHBS structures proved to be useful as a technology for in silico development of peptide aptamers. Based on this technology, a number of peptide sequences of 15-30 amino acid residues have been proposed, synthesized and their selectivity was experimentally tested with target proteins. This analysis was carried out for three proteins used as biomarkers of cardiovascular diseases: myoglobin, troponin, and myeloperoxidase [3,4]. The peptide sequences prepared for these proteins are presented in Table 1. Some of these peptides were tested by capillary electrophoresis-on-a-chip. Electropherograms of proteins, peptides and their mixtures show that when peptides bind to the target protein, the elution time shifts and the area of the aptamer zone decreases. 
Table 1. List of synthesized peptides.

\begin{tabular}{ccc}
\hline $\begin{array}{c}\text { Name of } \\
\text { Peptide }\end{array}$ & Structure & $\begin{array}{c}\text { Number of } \\
\text { Amino Acid Residues }\end{array}$ \\
\hline LETI-3 & LTALGGILKKKGHHEAELKPLAQSHATKHK & \\
LETI-4 & LTALGGILKKKGQHAAELKPLAQSHATKHK & 30 \\
LETI-5 & LTALGGILKKKGQHAAELKPLAQSAATKAK & \\
LETI-6 & LTALGGILKKKGQGAAELKPLAQSAATKAK & \multirow{2}{*}{38} \\
\hline LETI-2 & HLNEDQLREKAKELWQTIYNLEAEKFDLQEKFKQQKE & \\
LETI-7 & TLNEDQLREKAKELAQTIANLEAEKIDLQEKAKQQKYE & \multirow{2}{*}{} \\
\hline LETI-8 & SLMFMQWGQLLDHDLD & \\
LETI-8.1 & SLMLMQHGQLLDHDLD & \\
LETI-8.2 & SLMLMQNGQLLDHDLD & \\
LETI-8.3 & SLMSMQHGQLLDHDLD & \\
LETI-8.4 & SLMSMQNGQLLDHDLD & \\
\hline
\end{tabular}

The following requirements were put forward for the peptide aptamers being developed: complementarity to the target protein, high selectivity, and absence of background fluorescence. Therefore, the presented sequences have differences in the substitution of aromatic amino acids. The sequences shown were synthesized and tested by capillary electrophoresis-on-a-chip.

\section{Results and Discussion}

In this work, it is proposed to carry out direct detection of the fluorescence of biomarkers, selectively bound by peptide aptamers, without the use of special fluorescent labels. The latter are labile and expensive substances and their exclusion from the protocol can increase the shelf life and ease the tests, as well as reduce the cost of consumables. This is the novelty of the proposed technical solutions.

Protein fluorescence is excited in the ultraviolet range of spectrum $(220-280 \mathrm{~nm})$. Proteins contain three amino acid residues that can contribute to fluorescence: tyrosine (Tyr), tryptophan (Trp), and phenylalanine (Phe). Among them tryptophan demonstrates the highest quantum yield. About $90 \%$ of all protein fluorescence is usually due to tryptophan residues. This natural fluorophore is extremely sensitive to the polarity of the environment [5]. Spectral shifts are often the result of several phenomena, among which ligand binding, protein-protein association, and denaturation can be distinguished. In addition, the emission maxima of proteins reflect the average availability of their tryptophan residues in the aqueous phase. Proteins absorb light in the vicinity of $280 \mathrm{~nm}$, and the maxima of the fluorescence spectra lie in the region of $320-350 \mathrm{~nm}$. The decay times of the fluorescence of tryptophan residues are in the range of 1-6 ns. Tyrosine fluoresces intensively in solution, but its fluorescence is much weaker in the composition of proteins. The maximum absorption of tyrosine and tryptophan occurs at a wavelength of $280 \mathrm{~nm}$. Phenylalanine is excited at shorter wavelengths, and the quantum yield of phenylalanine in proteins is low, so the fluorescence of this amino acid residue is negligible in the proposed detection scheme. Protein absorption at 280 $\mathrm{nm}$ is associated with tyrosine and tryptophan residues.

Since the purpose of this work is to study the possibility of realizing on-a-chip a highly sensitive analysis of protein markers of acute and chronic diseases of the cardiovascular system without the use of fluorescent labels, and on the basis of characteristic fluorescence of protein molecules in the ultraviolet range of radiation, it is obvious that to achieve this goal, biorecognition elements (peptide aptamers) immobilized on the chip should have minimal background fluorescence. These is not applied to nucleotide aptamers, since they fluoresce when excited by blue and ultraviolet radiation. Therefore, it is advisable to investigate the possibility of using peptide aptamers, especially those not containing aromatic compounds.

The setup for fluorimetric analysis of protein structures is shown in Figure 1. It consists of three main elements: UV-LED, biochip, and a CMOS sensor. 


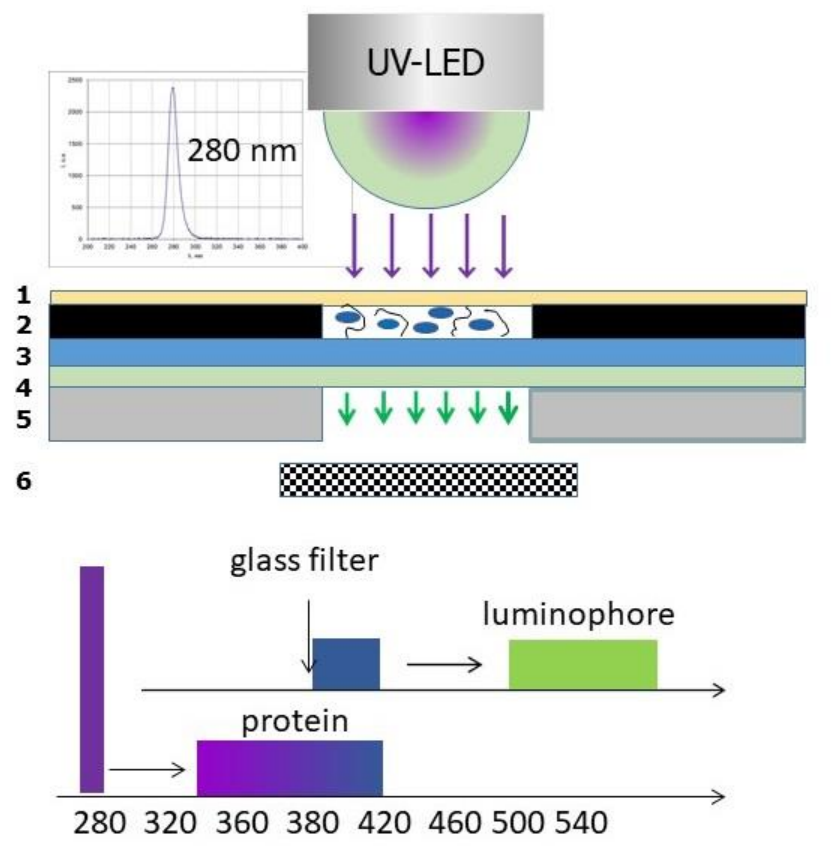

(a)

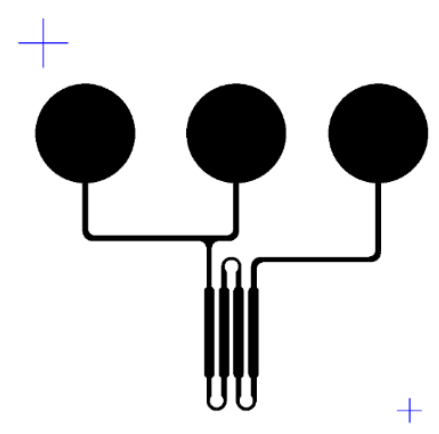

(b)

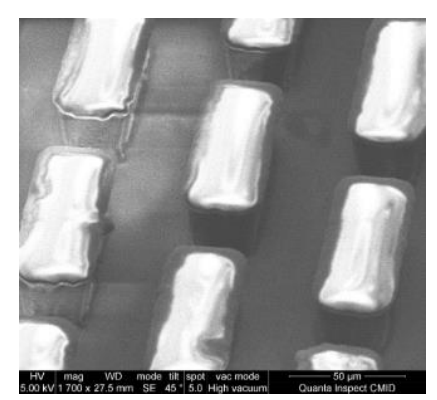

(c)

Figure 1. Setup for fluorimetric analysis of proteins: (a) Biochip structure: 1-polypropylene (thickness $100 \mu \mathrm{m}) ; 2-S U-8(100 \mu \mathrm{m}) ; 3$-glass $(0.2 \mathrm{~mm})$; 4-luminophore layer; 5-PMMA (1.5 mm); 6-CMOS sensor; (b) Topology of a microfluidic system with rectangular detection pads; (c) SEM image of the detection area of the microfluidic system.

A biochip (Figure 1a) is a sandwich structure, which consists of a polymer substrate with channels on which glass is installed with immobilized peptides that perform the function of molecular recognition of the target protein. A thin layer of luminophore is deposited on the outer surface of the glass. The sample flows in the microfluidic system to enter the active sites of the biochip, where the "target protein-peptide" complex is formed. The topology of the microfluidic biochip (Figure 1b) includes inlet and outlet openings for sample load and drain. An inlet opening is connected to a pump; microfluidic channels, and a detection area divided into 4 segments, on which various aptamers are immobilized. On these segments, islands of SU-8 photoresist were formed using photolithography (Figure 1c) to improve the stability of the immobilized peptides. The chip body is created using 3D printing technology. The luminophore layer was applied to the glass from the liquid phase. The LED is a source of ultraviolet (UV) radiation with a wavelength of $280 \mathrm{~nm}$, which excites the natural fluorescence of proteins, which is detected by a CMOS sensor. From the CMOS sensor, the image is displayed by the computer and then analyzed using special software.

\section{Conclusions}

In this paper, a biosensor system for label-free detection of protein markers of cardiovascular diseases based on peptide aptamers is presented. To simulate them in silico, the CIHBS technique was used, which opens up the possibility of expanding the panel of peptide ligands to create multiparametric biochips. A number of aptamers for troponin, myoglobin and myeloperoxidase have been developed and synthesized. The structure and topology of a multiparametric biochip capable of simultaneous detection of 4 disease markers is presented. The optimal topology of a planar microfluidic system is found that meets the basic requirements, and the most promising technology of formation is selected. The relief and morphology of the surface of the microfluidic system obtained using photolithography and SU-8 photoresist are investigated. The use of these technologies to create portable devices for express diagnostics will have a positive effect on the dynamics of morbidity, disability and mortality of the population because of diseases of the cardiovascular system. 
Author Contributions: Conceptualisation, N.S., T.Z.; methodology, T.Z., V.K.; software, V.K.; investigation, O.N., M.K.; writing - original draft preparation, N.S., T.Z.; writing - review and editing, N.S., T.Z.

Funding: The reported study was funded by RFBR, project number 19-32-90020.

Acknowledgments: Authors acknowledge with thanks A. Kolobov, D. Sci. (The State Research Institute of Highly Pure Biopreparations St. Petersburg, Russia) for synthesis of peptide aptamers.

Conflicts of Interest: The authors declare no conflicts of interest.

\section{References}

1. Sitkov, N.O.; Zimina, T.M.; Karasev, V.A.; Lemozerskii, V.E.; Kolobov, A.A. Design of Peptide Ligands (Aptamers) for Determination of Myeloperoxidase Level in Blood Using Biochips. In Proceedings of the 2020 IEEE Conference of Russian Young Researchers in Electrical and Electronic Engineering (EIConRus), St. Petersburg and Moscow, Russia, 27-30 January 2020; pp. 1599-1603.

2. Karasev, V. A model of molecular vector machine of proteins. BioSystems 2019, 180, 7-18.

3. Sitkov, N.O.; Karasev, V.A.; Luchinin, V.V.; Zimina, T.M. Development of biosensors for express-detection of protein markers of diseases in blood using peptide biorecognition elements. In AIP Conference Proceedings; AIP Publishing LLC: 2019; Volume 2140, p. 020072.

4. Sitkov, N.O.; Zimina, T.M.; Soloviev, A.V.; Lemozerskii, V.E.; Karasev, V.A. Development of Peptide Aptamers-3d Complementary Ligands for Selective Binding of Target Biomarkers of Diseases in Multiparametric Sensor Systems. In Proceedings of the 2019 IEEE Conference of Russian Young Researchers in Electrical and Electronic Engineering (EIConRus), Saint Petersburg and Moscow, Russia, 28-31 January 2019; pp. 1327-1330.

5. Lakowicz, Joseph R. Principles of Fluorescence Spectroscopy; Springer Science+Business Media: NY, USA, 2006; p. 954.

Publisher's Note: MDPI stays neutral with regard to jurisdictional claims in published maps and institutional affiliations.

(C) 2020 by the authors. Submitted for possible open access publication under the terms and conditions of the Creative Commons Attribution (CC BY) license (http://creativecommons.org/licenses/by/4.0/). 